\title{
Opposition based red wolf algorithm for solving optimal reactive power problem
}

\author{
Lenin Kanagasabai \\ Department of Electrical and Electronic Engineering, Prasad V. Potluri Siddhartha Institute of Technology, India
}

\begin{tabular}{l} 
Article Info \\
\hline Article history: \\
Received Feb 12, 2021 \\
Revised Apr 2, 2021 \\
Accepted Apr 24, 2021 \\
\hline
\end{tabular}

\section{Keywords:}

Opposition based learning Optimal reactive power Red wolf optimization Transmission loss

\begin{abstract}
This paper presents an opposition based red wolf optimization (ORWO) algorithm for solving optimal reactive power problem. Each red wolf has a flag vector in the algorithm, and length is equivalent to the whole sum of numbers which features in the dataset of the wolf optimization (WO). In this proposed algorithm, red wolf optimization algorithm has been intermingled with opposition-based learning (OBL). By this amalgamate procedure the convergence speed of the proposed algorithm will be increased. To discover an improved candidate solution, the concurrent consideration of a probable and its corresponding opposite are estimated which is closer to the global optimum than an arbitrary candidate solution. Proposed algorithm has been tested in standard IEEE 14-bus and 300-bus test systems. The simulation results show that the proposed algorithm reduced the real power loss considerably.
\end{abstract}

This is an open access article under the CC BY-SA license.

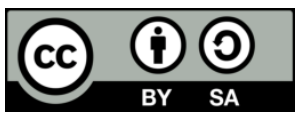

Corresponding Author:

Lenin Kanagasabai

Department of Electrical and Electronic Engineering

Prasad V. Potluri Siddhartha Institute of Technology

Kanuru, Vijayawada, Andhra Pradesh 520007, India

Email: gklenin@gmail.com

\section{INTRODUCTION}

In this work the key objective is actual power loss reduction. Optimal reactive power problem has been solved by a variety of methods [1]-[6]. However many technical hitches are found while solving problem due to an assortment of constraints. Evolutionary techniques [7]-[16] are applied to solve the reactive power problem, but the key problem is some algorithms stuck in local optimal solution, and failed to balance the exploration and exploitation during the search of global solution. This paper proposes an opposition based red wolf optimization (ORWO) algorithm for solving optimal reactive power problem. Each red wolf has a flag vector in the algorithm and length is equivalent to the whole sum of numbers which features in the dataset of the wolf optimization (WO) [17]-[19]. In this proposed algorithm red wolf optimization algorithm has been intermingled with opposition based learning (OBL) [20]. By this convergence speed will be increased. To discover an improved candidate solution, the concurrent consideration of a probable and its corresponding opposite are estimated which is closer to the global optimum than an arbitrary candidate solution. Proposed algorithm has been tested in standard IEEE 14-bus and 300-bus test systems. The simulation results show that the proposed algorithm reduced the real power loss considerably.

\section{PROBLEM FORMULATION}

Objective of the problem is to reduce the true power loss: 


$$
\mathbf{F}=\mathbf{P}_{\mathrm{L}}=\sum_{\mathrm{k} \in \mathrm{Nbr}} \mathbf{g}_{\mathrm{k}}\left(\mathrm{V}_{\mathrm{i}}^{2}+\mathrm{V}_{\mathrm{j}}^{2}-2 \mathbf{V}_{\mathrm{i}} \mathbf{V}_{\mathrm{j}} \cos \theta_{\mathrm{ij}}\right)
$$

Voltage deviation given as (2).

$$
F=P_{L}+\omega_{v} \times \text { Voltage Deviation }
$$

Voltage deviation given by (3).

$$
\text { Voltage Deviation }=\sum_{\mathrm{i}=1}^{\mathrm{Npq}}\left|\mathbf{V}_{\mathbf{i}}-\mathbf{1}\right|
$$

Equality constraint given by (4).

$$
\mathbf{P}_{\mathrm{G}}=\mathbf{P}_{\mathrm{D}}+\mathbf{P}_{\mathbf{L}}
$$

Inequality constraints are given by (5)-(9).

$$
\begin{aligned}
& \mathbf{P}_{\mathrm{gslack}}^{\min } \leq \mathbf{P}_{\mathrm{gslack}} \leq \mathbf{P}_{\mathrm{gslack}}^{\mathrm{max}} \\
& \mathbf{Q}_{\mathrm{gi}}^{\text {min }} \leq \mathbf{Q}_{\mathrm{gi}} \leq \mathbf{Q}_{\mathrm{gi}}^{\text {max }}, \mathbf{i} \in \mathbf{N}_{\mathrm{g}} \\
& \mathbf{V}_{\mathbf{i}}^{\text {min }} \leq \mathbf{V}_{\mathbf{i}} \leq \mathbf{V}_{\mathbf{i}}^{\text {max }}, \mathbf{i} \in \mathbf{N} \\
& \mathbf{T}_{\mathbf{i}}^{\text {min }} \leq \mathbf{T}_{\mathbf{i}} \leq \mathbf{T}_{\mathbf{i}}^{\text {max }}, \mathbf{i} \in \mathbf{N}_{\mathrm{T}} \\
& \mathrm{Q}_{\mathrm{c}}^{\min } \leq \mathrm{Q}_{\mathrm{c}} \leq \mathrm{Q}_{\mathrm{C}}^{\max }, \mathrm{i} \in \mathrm{N}_{\mathrm{C}}
\end{aligned}
$$

\section{OPPOSITION BASED RED WOLF OPTIMIZATION}

Red wolf optimization mimics the communal management and hunt deeds of red wolves in nature. There are three fittest candidate solutions assumed as $\alpha, \beta$ and $\gamma$ to lead the population toward promising regions of the exploration space in each iteration of red wolf optimization. $\varphi$ is named for the rest of red wolves and it will assist $\alpha, \beta$ and $\gamma$ to encircle, hunt, and attack prey, that is to find enhanced solutions. In order to scientifically replicate the encompassing behavior of red wolves, The (10) is proposed.

$$
\vec{G}=\left|\vec{F} \cdot \overrightarrow{Y_{P}}(t)-\vec{Y}(t)\right|, \vec{Y}(t+1)=\overrightarrow{Y_{P}}(t)-\vec{H} \cdot \vec{G}
$$

Where $t$ indicates the current iteration, $\overrightarrow{\mathrm{H}}=2 \overrightarrow{\mathrm{b}} \cdot \overrightarrow{\mathrm{r}_{1}}-\overrightarrow{\mathrm{b}}, \overrightarrow{\mathrm{F}}=2 \cdot \overrightarrow{\mathrm{r}_{2}}, \widehat{\mathrm{Y}_{\mathrm{P}}}$ the position vector of the prey, $\overrightarrow{\mathrm{Y}}$ is the position vector of a red wolf, $\vec{b}$ is linearly decreased from 2.0 to 0 , and $\overrightarrow{r_{1}}$ and $\overrightarrow{r_{2}}$ are arbitrary vectors in $[0,1]$. proposed.

In order to mathematically simulate the hunting behavior of red wolves, The (11), (12), and (13) are

$$
\begin{aligned}
& \overrightarrow{G_{\alpha}}=\left|\overrightarrow{F_{1}}, \overrightarrow{Y_{\alpha}}-\vec{Y}\right| \\
& \overrightarrow{G_{\beta}}=\left|\overrightarrow{F_{2}}, \overrightarrow{Y_{\beta}}-\vec{Y}\right| \\
& \overrightarrow{G_{\gamma}}=\left|\overrightarrow{F_{3}}, \overrightarrow{Y_{\gamma}}-\vec{Y}\right| \\
& \overrightarrow{Y_{1}}=\overrightarrow{Y_{\alpha}}-\overrightarrow{H_{1}} \cdot \overrightarrow{G_{\alpha}} \\
& \overrightarrow{Y_{2}}=\overrightarrow{Y_{\beta}}-\overrightarrow{H_{2}} \cdot \overrightarrow{G_{\beta}} \\
& \overrightarrow{Y_{3}}=\overrightarrow{Y_{\gamma}}-\overrightarrow{H_{3}} \cdot \overrightarrow{G_{\gamma}} \\
& \vec{Y}(t+1)=\frac{\overrightarrow{Y_{1}}+\overrightarrow{Y_{2}}+\overrightarrow{Y_{3}}}{3}
\end{aligned}
$$

The position of a red wolf was updated by (13) and (14) is used to discrete the position.

$$
\text { flag }_{i, j}= \begin{cases}1 & Y_{i, j}>0.50 \\ 0 & \text { otherwise }\end{cases}
$$

Where $\mathrm{i}$, indicates the jth position of the ith red wolf, $\operatorname{flag}_{i, j}$ is features of the wolf. 
OBL is one of the influential optimization tools to boost the convergence speed of different optimization techniques. The thriving implementation of the OBL engages evaluation of opposite population and existing population in the similar generation to discover the superior candidate solution of a given reactive power problem. The conception of opposite number requirements is to be defined to explain OBL. Let $N(N \in[x, y])$ be a real number and the $N^{o}$ (opposite number) can be defined as (15).

$$
N^{o}=x+y-N
$$

In the exploration space it has been extended as (16).

$$
N_{i}^{o}=x_{i}+y_{i}-N_{i}
$$

Where $\left(N_{1}, N_{2}, \ldots N_{d}\right)$ is a point in the dimensional search space, $N_{i} \in\left[x_{i}, y_{i}\right], i \rightarrow\{1,2,3, \ldots d\}$

In all oppositional based optimization, the conception of OBL is used in the initialization procedure and as well as in each iteration using the generation jumping rate, Jr.

Step 1: The positions of red wolves (search agents) are initialized arbitrarily in the exploration space. Population size (number of wolves) and number of iterations are fixed.

Step 2: Compute fitness value of each search agent which represents the distance of wolf from the prey.

Step 3: Initialize opposite points and use them to generate opposite population and compute the fitness of each individual populations.

Step 4: Perform sorting among existing population (pop) and opposite population (opop), based on their fitness values.

Step 5: Select $\mathrm{nP}$ number of fittest solutions from the combination of the existing and the equivalent opposite population.

Step 6: Based on the fitness values, solutions are identified which represent the alpha, beta and delta category wolves, respectively.

Step 7: Modify the positions of the red wolves

Step 8: Update the fitness value using the modified position of the Red wolves.

Step 9: Using jumping rate, the opposite population are generated from the current population.

Step 10: Select nP number of fittest solutions from the combination of the current and the opposite population.

Step 11: Repeat step 6 to step 10 until the maximum number of iterations is reached.

Step 12: Output the best solution.

The pseudocode is shown in Algorithm 1.

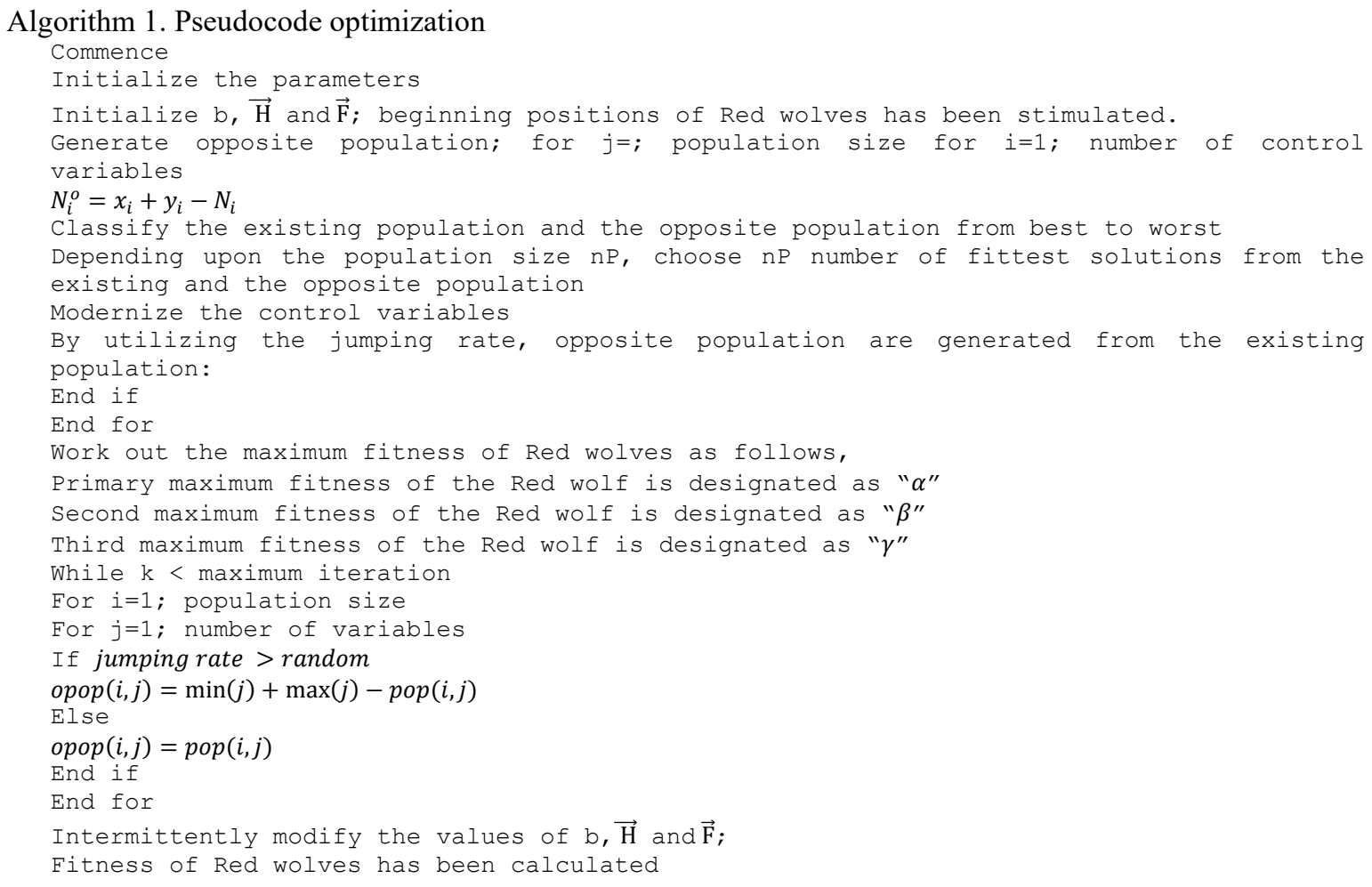




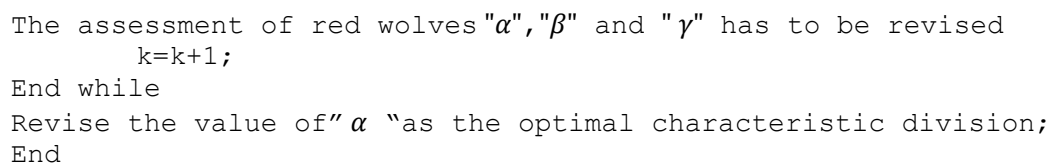

\section{SIMULATION RESULTS}

At first in standard IEEE 14-bus system the validity of the proposed ORWO algorithm has been tested and comparison results are presented in Table 1. Figure 1 provides the details of comparison of real power loss. Then IEEE 300-bus system [22] is used as test system to validate the performance of the ORWO algorithm. Table 2 shows the comparison of real power loss obtained after optimization. Compared to EEA method, the proposed ORWO reduces $5.58 \%$ real power loss. Real power loss has been considerably reduced when compared to the other standard reported algorithms.

Table 1. Comparison results

\begin{tabular}{cccc}
\hline Control variables & ABCO [21] & IABCO [21] & Proposed ORWO \\
\hline V1 & 1.06 & 1.05 & 1.02 \\
V2 & 1.03 & 1.05 & 1.01 \\
V3 & 0.98 & 1.03 & 1.00 \\
V6 & 1.05 & 1.05 & 1.00 \\
V8 & 1.00 & 1.04 & 0.90 \\
Q9 & 0.139 & 0.132 & 0.100 \\
T56 & 0.979 & 0.960 & 0.900 \\
T47 & 0.950 & 0.950 & 0.900 \\
T49 & 1.014 & 1.007 & 1.000 \\
Ploss (MW) & 5.92892 & 5.50031 & 4.54826 \\
\hline
\end{tabular}

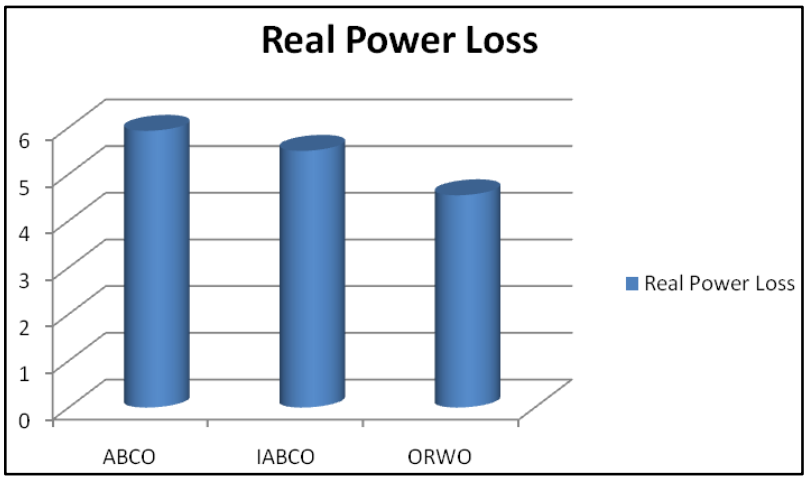

Figure 1. Comparison of real power loss

Table 2. Comparison of real power loss

\begin{tabular}{ccccc}
\hline Parameter & EEA Method [23] & EGA Method [23] & CSA Method [24] & Proposed ORWO \\
\hline PLOSS (MW) & 650.6027 & 646.2998 & 635.8942 & 614.2846 \\
\hline
\end{tabular}

\section{CONCLUSION}

ORWO algorithm has successfully solved the reactive power optimization problem. In this proposed ORWO algorithm has been intermingled with OBL. By this amalgamate procedure the convergence speed of the proposed algorithm has been increased. Proposed algorithm has been tested in standard IEEE 14-bus and 300-bus test systems. Simulation results show that the proposed ORWO algorithm reduced the real power loss considerably. Compared to EEA method, the proposed ORWO has reduced $5.58 \%$ real power loss.

\section{REFERENCES}

[1] K. Y. Lee, Y. M. Park, and J. L. Ortiz, "Fuel-cost minimisation for both real and reactive-power dispatches," Proceedings Generation, Transmission and Distribution Conference, vol. 131, no. 3, pp. 85-93, 1984. 
[2] N. I. Deeb and S. M. Shahidehpour, "An efficient technique for reactive power dispatch using a revised linear programming approach," Electric Power System Research, vol. 15, no. 2, pp. 121-134, 1988.

[3] M. R. Bjelogrlic, M. S. Calovic, P. Ristanovic and B. S. Babic, "Application of Newton's optimal power flow in voltage/reactive power control," IEEE Trans Power System, vol. 5, no. 4, pp. 1447-1454, 1990.

[4] S. Granville, "Optimal reactive dispatch through interior point methods," IEEE Transactions on Power System, vol. 9, no. 1 , pp. 136-146, 1994.

[5] N. Grudinin, "Reactive power optimization using successive quadratic programming method," IEEE Transactions on Power System, vol. 13, no. 4, pp. 1219-1225, 1998.

[6] W. Yan, J. Yu, D. C. Yu and K. Bhattarai, "A new optimal reactive power flow model in rectangular form and its solution by predictor corrector primal dual interior point method," IEEE Transactions on power systems, vol. 21, no. 1, pp.61-67, 2006.

[7] A. Mukherjee and V. Mukherjee, "Solution of optimal reactive power dispatch by chaotic krill herd algorithm," IET Generation, Transmission \& Distribution, vol. 9, no. 15, pp. 2351-2362, 2015.

[8] M. A/P Morgan, N. R. H. Abdullah, M. H. Sulaiman, M. Mustafa and R. Samad, "Computational intelligence technique for static VAR compensator (SVC) installation considering multi-contingencies (N-m)," ARPN Journal of Engineering and Applied Sciences, vol. 10, no. 22, pp. 17059-17064, 2015.

[9] M. H. Sulaiman, Z. Mustaffa, H. Daniyal, M. R. Mohamed and O. Aliman, "Solving optimal reactive power planning problem utilizing nature inspired computing techniques," ARPN Journal of Engineering and Applied Sciences, vol. 10, no. 21, pp. 9779-9785, 2015.

[10] M. H. Sulaiman, W. L. Ing, Z. Mustaffa and M. R. Mohamed, "Grey wolf optimizer for solving economic dispatch problem with valve-loading effects," ARPN Journal of Engineering and Applied Sciences, vol. 10, no. 21, pp. 9796-9801, 2015.

[11] K. Pandiarajan, and C. K. Babulal, "Fuzzy harmony search algorithm based optimal power flow for power system security enhancement". International Journal of Electrical Power \& Energy Systems, vol. 78, pp. 72-79. 2016.

[12] M. H. Sulaiman, Z. Mustaffa, M. R. Mohamed and O. Aliman, "An application of multi-verse optimizer for optimal reactive power dispatch problems," International Journal of Simulation: Systems, Science and Technology, vol. 17, no. 41, pp. 5.1-5.5. 2017.

[13] M. A/P Morgan, N. R. H. Abdullah, M. H. Sulaiman, M. Mustafa and R. Samad, "Multi-objective evolutionary programming (MOEP) using mutation based on adaptive mutation operator (AMO) applied for optimal reactive power dispatch," ARPN Journal of Engineering and Applied Sciences, vol. 11, no. 14, pp. 8884-8888, 2016.

[14] R. Ng Shin Mei, M. H. Sulaiman, Z. Mustaffa, "Ant lion optimizer for optimal reactive power dispatch solution," Journal of Electrical Systems, "Special Issue AMPE2015", pp. 68-74, 2016.

[15] M. Morgan, N. R. H. Abdullah, M. H. Sulaiman, M. Mustafa, R. Samad, "Benchmark studies on optimal reactive power dispatch (ORPD) based multi-objective evolutionary programming (MOEP) using mutation based on adaptive mutation adapter (AMO) and polynomial mutation operator (PMO)," Journal of Electrical Systems, vol. 12, no. 1, pp. 121-132, 2016.

[16] R. Ng Shin Mei, M. H. Sulaiman, Z. Mustaffa, H. Daniyal, "Optimal reactive power dispatch solution by loss minimization using moth-flame optimization technique," Applied Soft Computing, vol. 59, pp. 210-222, 2017.

[17] S. Mirjalili, S. M. Mirjalili, A. Lewis, "Grey wolf optimizer," Advances in engineering software, vol. 69, pp. 4661, 2014.

[18] El-Fergany, A. Attia, H. M. Hasanien, "Single and multi-objective optimal powerflow using grey wolf optimizer and differential evolution algorithms," Electric Power Components and Systems, vol. 43, no. 13, pp. 1548-59, 2015.

[19] M. R. Shakarami and I. Faraji, "Design of SSSC-based stabilizer to damp inter-area oscillations using gray wolf optimization algorithm," SID: Conference on Power Plants, 2015, pp. 1-8.

[20] Tizhoosh H R. "Opposition-based learning: a new scheme for machineintelligence. In: Proceeding of the international conference on computationalintelligence for modeling, control and automation," International conference on computational intelligence for modelling, control and automation and international conference on intelligent agents, web technologies and internet commerce (CIMCA-IAWTIC'06), vol. 1, 2005, pp. 695-701.

[21] C. M. K. Sivalingam, S. Ramachandran and P. S. S. Rajamani, "Reactive power optimization in a power system network through metaheuristic algorithms," Turkish Journal of Electrical Engineering \& Computer Science, vol. 25, no. 6, pp. 4615 - 4623, 2017. Doi: 10.3906/elk-1703-159.

[22] IEEE, "The IEEE-test systems," 1993, [Online]. Available: https://electricgrids.engr.tamu.edu/electric-grid-testcases/ieee-300-bus-system/.

[23] S. S. Reddy, P. R. Bijwe and A. R. Abhyankar, "Faster evolutionary algorithm based optimal power flow using incremental variables," Electrical Power and Energy Systems, vol. 54, pp. 198-210, 2014.

[24] S. S. Reddy, "Optimal reactive power scheduling using cuckoo search algorithm," International Journal of Electrical and Computer Engineering, vol. 7, no. 5, pp. 2349-2356, 2017. 